\title{
H $\alpha$ FINE STRUCTURE AND THE DYNAMICS OF THE SOLAR ATMOSPHERE
}

FRANZ-LUDWIG DEUBNER

Fraunhofer Institut, Freiburg, G.F.R.

\begin{abstract}
Series of simultaneous spectra in different spectral regions, representing all 'visible' layers of the solar atmosphere, of high spatial and temporal resolution are used to compute power spectra and diagnostic diagrams of intensity and velocity fluctuations in order to study the dynamic response of the chromosphere to disturbances from below.

Although these spectra provide the highest spatial resolution presently available, most of the oscillatory power appears to be concentrated towards relatively low wavenumbers corresponding to wavelengthes of $12^{\prime \prime}$. According to the periods observed, these waves must be evanescent ones. Therefore, internal gravity waves can be ruled out as a model for the 300 -s oscillations, whereas the observations fit well with the diagnostic data predicted by Ulrich, Leibacher and, more recently, Wolff on the basis of their models of trapped subphotospheric sound waves leaking into the photosphere.
\end{abstract}

Nevertheless, on closer inspection of the raw, un-transformed spectral data (cf. Figure 1) one finds sufficiently well isolated events, which exhibit in detail the different features predicted by the 'local disturbance' theories: acoustic modes in a 'nearby zone' about 2" in diameter, and internal gravity waves spreading further away from the center of disturbance. The energy involved in these events leaves no detectable traces in the diagnostic diagrams. Whether it merges into the larger scale 300 -s oscillation energy remains unclear.

The observational material being completed by simultaneous slit jaw pictures (K-line and white light), it is possible to study the evolution of structures under the slit to some extent in three dimensional space. It is shown how a disturbance, starting as a 'granular ring' in the photosphere, propagates upward (cf. Figure 1 and 2) to the top of the chromosphere, where a 'spicule' is formed.

The non-uniform structure of the magnetic field plays an important role in this process.

At the borders of a supergranule, where the magnetic fields are usually strong and vertical, the rising material is guided upward and kept together or even concentrated by the surrounding field lines. Away from the supergranular network the chromospheric magnetic field is either bent in different directions (as the fine structure elements become more and more elongated) or generally too weak to govern the fluid motions.

In the first case the disturbance will be deflected, maybe still visible as a mottle, but in most cases not visible in the plane of the spectragraph slit. In the second case the disturbance will, from the photosphere, propagate freely in all directions (instead of one) and will, therefore, be not powerful enough to eject a spicule. 


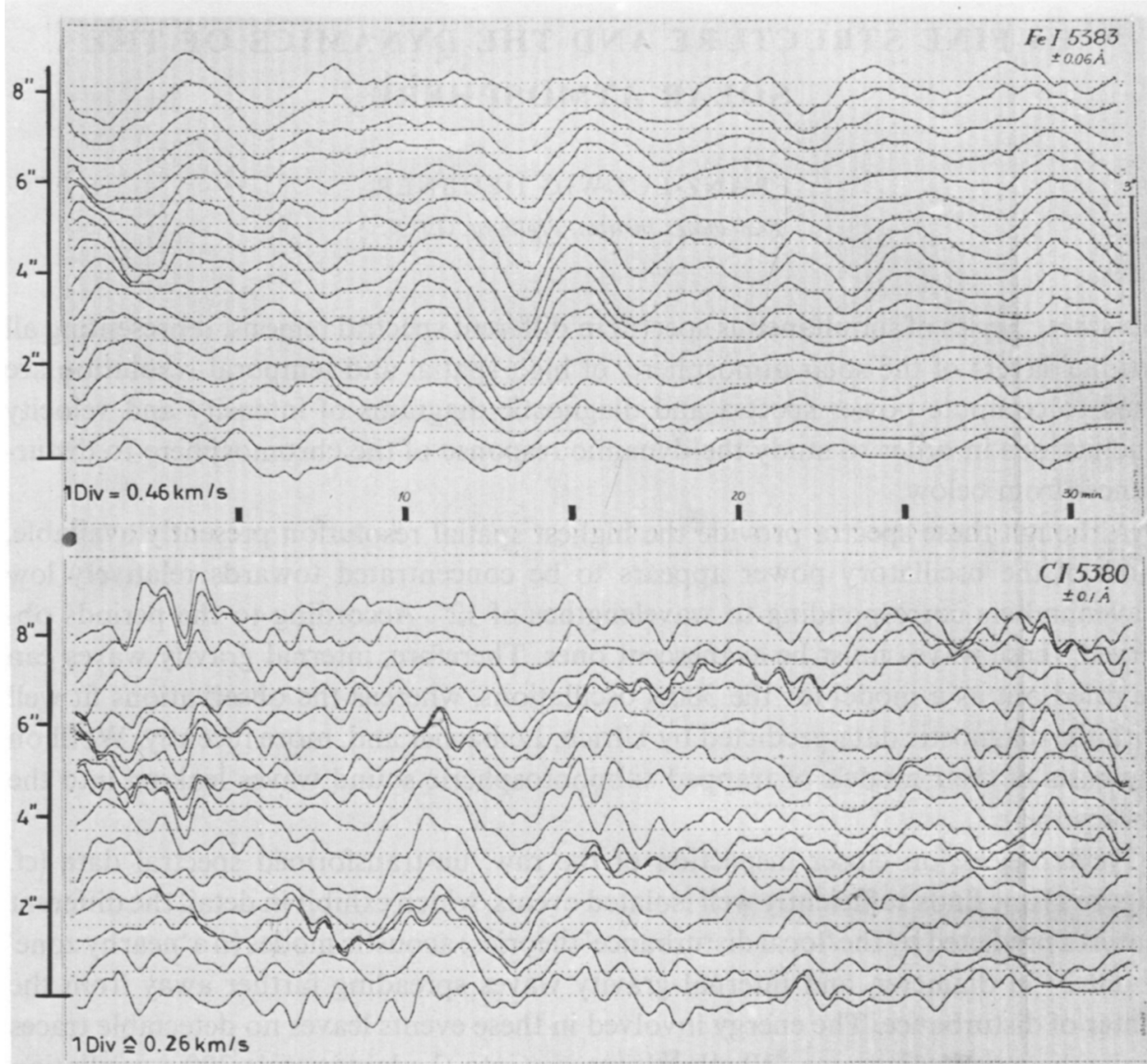

1 Div $\cong 0.26 \mathrm{~km} / \mathrm{s}$

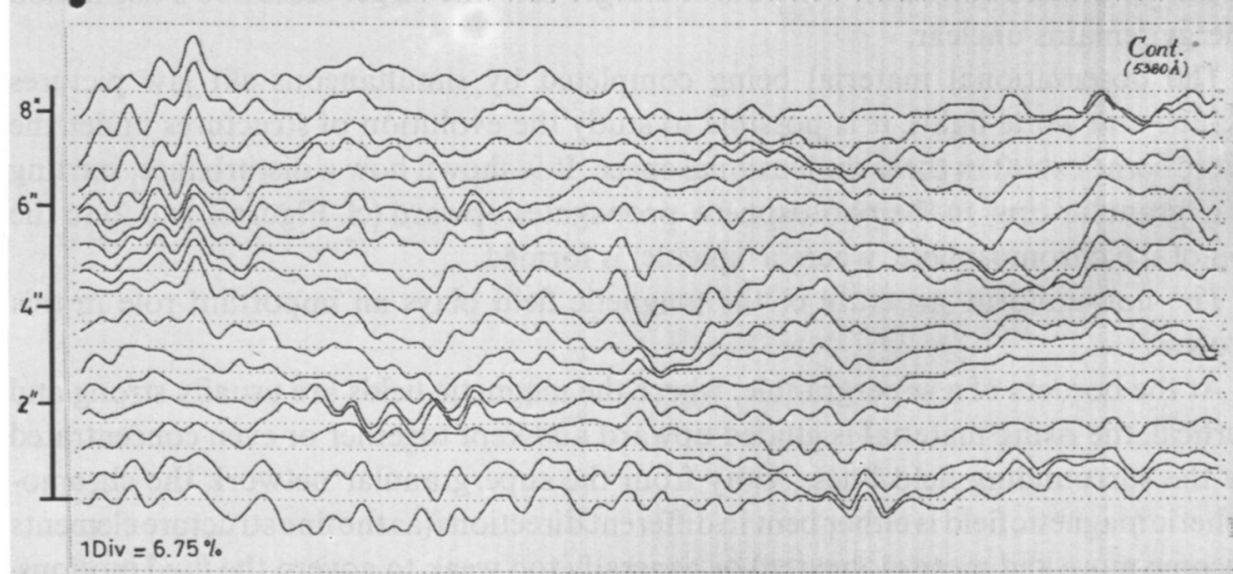

Fig. 1.

Fig. 1-2. Continuum intensity and velocities derived from line shift measurements in photospheric and chromospheric lines of increasing height of formation. The relevant scales are given in the figure. A photospheric disturbance is shown, which starts $\sim 9 \mathrm{~min}$ after the beginning of the observations, propagates through all layers of the atmosphere exciting various oscillating modes in the lower chromosphere and finally produces in $\mathrm{H} \alpha$ an abruptly rising spicular structure. 

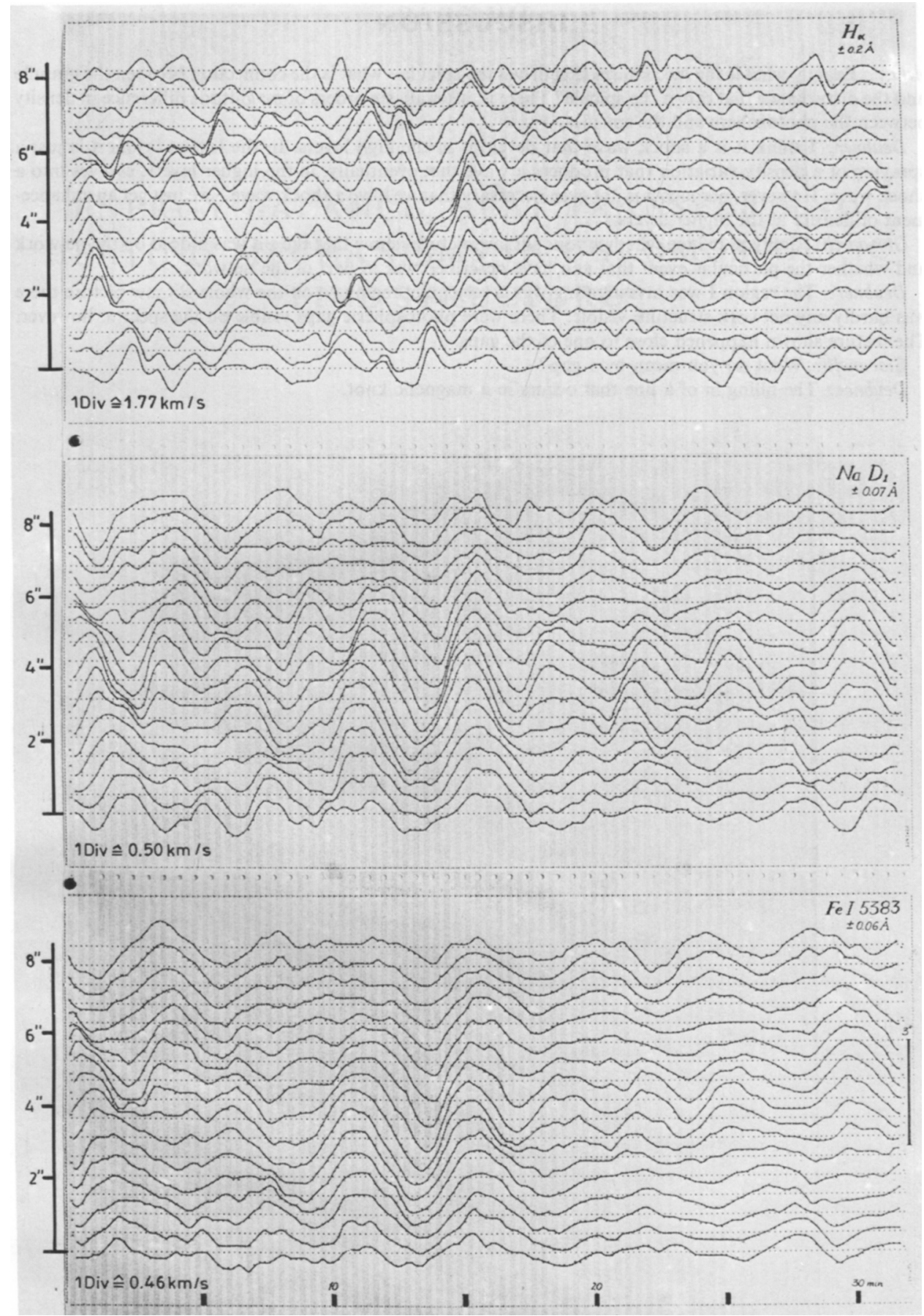

Fig. 2. 


\section{DISCUSSION}

Athay: Exactly what is the mechanism that drives the spicule - what is the connection between the granule and the disturbance that drives the spicule? There is, after all, six orders of magnitude difference in density between the photosphere and the spicules.

Deubner: I think it is a shock wave that develops in the high layers. In the lowest layers it is just a spraying of a density variation that produces a wave that eventually, in the higher layers, evolves into a shock wave. However, perhaps it is not even necessary that we have a shock wave - we just get an enhancement of density at the higher layers.

Giovanelli: Can you tell me whether your slit jaw pictures show that the slit was indeed on the network and whether the particular event that you have described was in part of the network.

Deubner: The region I was investigating was a very quiet region so on the whole slit jaw picture there was hardly any network structure visible. There were photospheric gaps visible on the spectra, however. The feature shown happened close to one of the gaps.

Giovanelli: What do you mean by a gap?

Deubner: The filling in of a line that occurs in a magnetic knot. 